in polarization-maintaining optical fibers', J. Lightwave Technol., 1983, LT-1, pp. 332-339

8 SUdARSHANAM, v. S., and SRINIVASAN, K.: 'Static phase change in a fiber optic coil hydrophone', Appl. Opt., 1990, 29, pp. 855-863

9 BUDIANSKY, B., DRUCKER, D. C.. KINO, G. S., and RICE, J. R : 'PressuTe BUDIANSKY, B., DRUCKER, D. C., KINO, G. S., and RICE, J. R.: 'Pressure
sensitivity of a clad optical fiber', Appl. Opt., 1979, 18, pp. 40854088

10 timoshinko, S. P., and Goodier, J. N.: 'Theory of elasticity' (McGraw-Hill, New York, 1970)

11 LaGakos, N., JaRzYNSKi, J., COLE, J. H., and buCaro, J. A.: 'Frequency and temperature dependence of elastic moduli of polymers', J. Appl. Phys., 1986, 59, pp. 4017-4031

\section{OPTO-ELECTRONIC HIGH ORDER FEEDBACK NEURAL NETWORK}

Indexing terms: Opto electronics, Neural networks, Nonlinear systems

A novel neural network design, in which nonlinearities are created by feedback, is described. It is called the HOFNET. The design is suitable for optical implementation because it is tolerant of the limited dynamic ranges present in optical systems. An optical system with electronic feedback was constructed and its operation is described.

There has been recent interest in the use of neural networks for the task of pattern recognition. In particular, neural networks with higher order nonlinearities have been shown to have substantially increased memory capacity over their lower order counterparts ${ }^{1}$ having the same number of neurons. Optical designs have been proposed ${ }^{1-4}$ but only a few second order optical networks have been constructed. The use of optical hardware offers the potential of large numbers of fully interconnected neurons operating at high speed. Optical systems suffer from a limited dynamic range, typically less than $30 \mathrm{~dB}$, because of saturation limits and high background noise levels. So signal levels emerging from a single component having a high order nonlinearity either run into saturation or are perturbed by the high input noise, in both cases losing valuable magnitude information. The higher the order, the worse the problem, although the greater memory capacity can overcome this problem.

In this letter we describe a different approach in which nonlinearities are created in an otherwise linear network by feedback. This High Order Feedback NET, or HOFNET, consists of $M$ parallel channels, where $M$ is the number of known exemplar patterns stored in the learning procedure. Each channel acts as an individual correlator ${ }^{5}$ which correlates the input pattern, $p$, with one of the stored patterns, $s_{i}$ $(i=1, \ldots, M)$. Every channel (Fig. 1) performs the same processing functions starting with the multiplication of an input constant signal level, $A$, by the Fourier transform of one of the known stored patterns, FT $\left(s_{i}\right)=S_{i}$. The output $A S_{i}$ is inverse Fourier transformed to give $A s_{i}$ which is then multiplied by the complex conjugate of the input pattern, $p$, which for real input patterns is just $p$ itself. This is followed by a further Fourier transform which, by the convolution theorem, yields the required correlation (denoted by *). Finally the correlation passes through an amplifier of gain $g$ to give $A g\left(S_{i}{ }^{*} P\right)$ which is fed back to the start of the loop to continue similar iterations. The output, taken from before the multiplication by the input pattern, is given on repeated iterations by $A s_{i}, A\left[g\left(S_{i}^{*} P\right)\right] s_{i}$, $A\left[g\left(S_{i}^{*} P\right)\right]^{2} . s_{i}, \ldots, A\left[g \cdot\left(S_{i}^{*} P\right)\right]^{n} . s_{i}$ after $n$ iterations. The order of the nonlinearity of the network is not fixed but

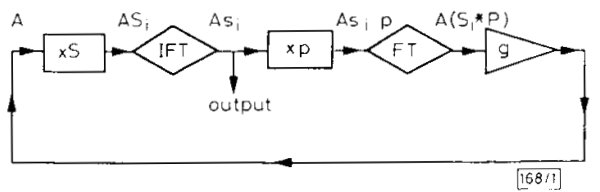

Fig. 1 Single channel of HOFNET increases by unity on each iteration until the strongest signal reaches saturation. Each iteration performs an independent calculation of the correlation which is multiplied by the product of all of the earlier similar calculations circulating in the loop. Time varying noise within the net or on the input pattern tends to be averaged out. The loop gain is given by $G=g\left(S_{i}^{*} P\right)$. If $G$ is unity the signal circulates at constant amplitude. If $G$ is greater than unity the signal magnitude increases as the loop iterates, increasing at higher rates the closer the input pattern is to the known stored pattern in that channel. If $G$ is less than unity the signal in that channel decreases, ultimately vanishing. The value of $G$ (and hence $g$ ) determines the radius of attraction of the net around each of the stored patterns. By setting $G$ correctly we can arrange that only input patterns sufficiently similar to the known stored pattern in the channel persist, circulating within the loop.

To see this clearly consider the case when the known stored patterns (having $N$ pixels or bits) are all $N$ of the members of a binary $(+1,-1)$, orthogonal set so that they are all equal Hamming distances apart. If the input, also assumed to be binary $(+1,-1)$, is less than $N / 4$ bits different from a given pattern, $s_{1}$, say, then the correct answer will be obtained if it converges to $s_{1}$. If it is more than $N / 4$ bits different, it will be closer to another of the orthogonal stored patterns and so it should not converge to $s_{1}$. Therefore, we need to set the radius of attraction to be $N / 4$ bits. Such an input would give a correlation peak with the pattern $s_{1}$ of $N / 2$. So we want patterns with correlations greater than $N / 2$ to persist and those less to vanish. This can be achieved by setting $\left(S_{i}^{*} P\right)=$ $N / 2$ for $G=1$ giving a fixed gain of $g=2 / N$. If any binary input pattern is put into the net each channel will iterate but only one pattern will persist. All others will decay to zero on sufficient repeated iterations so giving the correct answer.
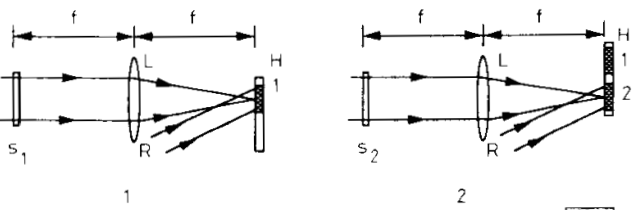

Fig. 2 Fourier transform hologram recording

The memory of our opto-electronic implementation of the HOFNET is provided by a spatially multiplexed Fourier transform hologram which is recorded during the training phase as shown in Fig. 2. The training exemplar pattern is illuminated by a HeNe laser through a ground glass screen to randomise the phase. This object is then Fourier transformed by a lens to give a Fourier transform hologram when interfered with a coherently related plane collimated reference beam on the recording plate. The ground glass screen produces an almost uniform intensity distribution in the Fourier transform which enables higher efficiency holograms to be recorded on the plate. The recording plate is moved laterally and further training patterns are recorded side by side in a square array on the plate. The hologram is formed in silver halide and bleached to give a phase hologram.

The opto-electronic HOFNET (Fig. 3) was designed so that the same input pattern would appear in each of the channels. This was achieved by allowing the channels to intersect at the input pattern while maintaining the individual identity of each channel by angular multiplexing. This has the advantage that noise on the input pattern is exactly transferred into each channel so no discrepancies between channels due to nonuniform or noisy input devices can occur.

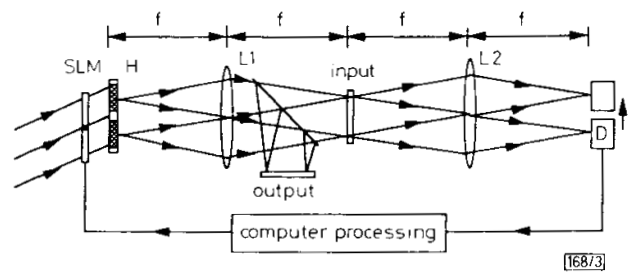

Fig. 3 Opto-electronic implementation of HOFNET

ELECTRONICS LETTERS 8th November 1990 Vol 26 No. 23 
The holographic store $(\mathrm{H})$ is replayed by a broad reference beam. The outputs from each hologram are Fourier transformed by a lens so that the original training patterns are reconstructed. This configuration causes the reconstructed images to be coincident in one plane. The input transparency is placed in this plane. Each reconstructed training pattern is multiplied by the input as they pass through. Thereafter the patterns emerging from the input transparency are Fourier transformed by a second lens to give the correlation on an optical detector array. In our initial experiment a single detector preceded by a pinhole was moved to detect the corre lations. The electronic detector output was fed back through an IBM XT computer to control the intensity of the light incident on each hologram independently by means of a liquid crystal spatial light modulator. In the experiment three orthogonal training patterns were stored. Each 16 pixel $(4 \times 4)$ binary pattern was reproduced twice, side by side, one copy being made the negative of the other, ${ }^{2}$ because of the difficulty of representing +1 and -1 in optics. The actual patterns consisted of $8 \times 4$ arrays. When one quarter of an input pattern was blocked the net converged to the complete correct pattern in two iterations.

In conclusion, the HOFNET has several benefits. The nonlinearity is produced by means of feedback and not by optical material nonlinearities and so the network is not limited by the switching speed, non-uniformity or availability of large arrays of opto-electronic nonlinear neurons. The feedback enables the HOFNET to be remarkably tolerant to input and system generated time varying noise and so makes it ideally suited for optical implementation.

The authors thank S. Q. Tao for recording the holograms, STC Technology Ltd., for donating the spatial light modula tor and the SERC, UK, funded Optoelectronic Interdisciplinary Research Centre for financial support.

D. R. SELVIAH

Itth September 1990

Z. Q. MAO

J. E. MIDWINTER

Department of Electronic and Electrical Engineering

University College, Torrington Place

London WC1E 7JE, United Kingdom

\section{References}

1 OWECHKo, Y., DUNNING, G. D., MAROM, E., and SOFFer, B. H.: 'Holographic associative memory with non-linearities in the correlation domain', Appl. Opt., 1987, 26, pp. 1900-1910

2 ATHALE, R. A., SZU, H. H., and FrIEDLANDER, c. B.: 'Optical implementation of associative memory with controlled nonlinearity in the correlation domain', Opt. Lett., 1986, 11, pp. 482-484

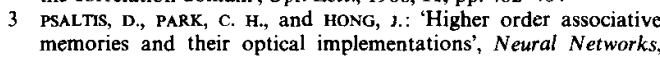
1988,1 , pp. $149-163$

4 JANG, J., SHIN, S., and LEE, s.: 'Programmable quadratic associative memory using holographic lenslet arrays', Opt. Lett., 1989, 14, pp. 838-840

5 SELVIAH, D. R., MIDWINTER, J. E., RIVERS, A. W., and LUNG, K. W. 'Correlating matched filter model for analysis and optimisation of neural networks', IEE Proc. F, 1989, 136, (3), pp. 143-148

\section{HIGH POWER ( $>100 \mathrm{~mW}$ ). LOW CROSSTALK $(<0.4 \%)$, INDIVIDUALLY ADDRESSED LASER DIODES}

Indexing terms: Semiconductor lasers, Diodes

Individually addressed high power dual channel diode lasers have been fabricated on $50 \mathrm{~mm}$ centres and bonded in a p-side up configuration. The diodes emit in a single spatial mode at up configuration. The diodes emit in a single spatial mode a greater than $100 \mathrm{~mW} \mathrm{CW}$ output power, and have a total
measured crosstalk of as low as $0.37 \%(-24 \mathrm{~dB})$ at $250 \mathrm{~Hz}$, measured crosstalk of
and $-33 \mathrm{~dB}$ at $1 \mathrm{MHz}$.

HIgh power, single element, coherent laser diodes have rapidly found application in communication, printing, and optical recording systems. In order to further increase the speed of these systems, it is desirable to integrate several such lasers monolithically ${ }^{-5}$ As a result, high speed printing and optical write/verify operations can be performed in parallel without significantly increasing the complexity of the optical system. The application requirements for the diode array then become: (i) individual addressability with close centre-tocentre spacing $(<150 \mu \mathrm{m})$, (ii) $>30 \mathrm{~mW}$ per emitter in a single spatial and spectral mode (for optical recording), and (iii) low crosstalk $(<2-4 \%)$ between emitters.

In this letter, we describe our results from high power individually-addressed dual channel laser diodes. Each diode is a $4 \mu \mathrm{m}$ wide, real refractive index-guided laser which emits in a single spatial mode to high power. ${ }^{6}$ The two-diode arrays are mounted $p$-side up onto a copper heat sink for $\mathrm{CW}$ testing, with individual wire bonds made to each $p$-each electrode. As described below, our results demonstrate that highpower, closely-spaced, low-crosstalk, reliable laser diode arrays can be fabricated in a $p$-up configuration, in which individual addressability is simple.

Fig. 1 shows the light-current characteristics of an array with diodes on $50 \mu \mathrm{m}$ centres, and with rear facet/front facet reflectivities of $95 \%$ and $5 \%$, respectively. Curves are shown for each array channel addressed separately, and for the two diodes when connected with a common contact. When separately addressed, each diode emits close to $140 \mathrm{~mW}$ in a single spatial mode, without adverse thermal effects. The spectrum consists of a single longitudinal mode near $830 \mathrm{~nm}$ wavelength to powers exceeding $100 \mathrm{~mW}$. When operated simultaneously, the total power exceeded $170 \mathrm{~mW}$ before the onset of thermal rollover.

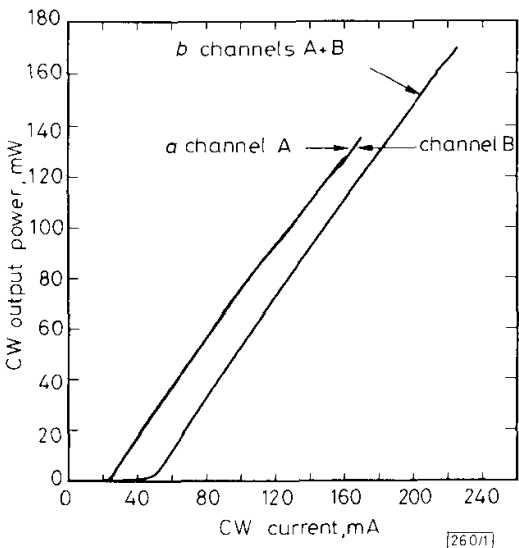

Fig. $1 \mathrm{CW}$ light-current characteristic of two-element individually addressed laser dio

a Channels $\mathrm{A}$ and $\mathrm{B}$ driven independently

$b$ Both channels driven in parallel. The diodes are on $50 \mu \mathrm{m}$ centres, and have front and rear facet reflection coatings of $5 \%$ and $95 \%$, respectively. HR $\lambda / 4$ coatings

The junction temperature rise, in addition to limiting the output power from the diode pair, contributes to the crosstalk between channels. The total crosstalk has three sources: thermal, electrical and optical. The relative importance of thermal and electrical components will largely be determined by the frequency of the laser operation. We have measured the total crosstalk at different frequencies to differentiate between the thermal and electrical components (for diodes on $50 \mu \mathrm{m}$ centres the optical crosstalk is below the measurement capability of our system).

At low frequencies, the crosstalk is dominated by thermal interaction. To measure thermal crosstalk, channel $\mathbf{A}$ is biased at $30 \mathrm{~mW}$ with a low-noise, DC current supply. Channel $\mathbf{B}$ is pulsed from zero current to $30 \mathrm{~mW}$ with $2 \mathrm{~ms}$ pulse lengths at a $50 \%$ duty cycle. Within the $2 \mathrm{~ms}$ pulse length, the diode array reaches thermal equilibrium. The steady-state sag in the optical output of channel A caused by the operation of channel $B$ is measured by imaging the near field of channel $A$ onto a $\mathrm{Si}$ photodetector. The drop in the optical output of channel $A$, as a percentage of its output with $B$ off, is the total 